\title{
The use of brain imaging to elucidate neural circuit changes in cocaine addiction
}

\author{
This article was published in the following Dove Press journal: \\ Substance Abuse and Rehabilitation \\ II September 2012 \\ Number of times this article has been viewed
}

\author{
Colleen A Hanlon ${ }^{1,2}$ \\ Melanie Canterberry' \\ 'Department of Psychiatry and \\ Behavioral Sciences, ${ }^{2}$ Department of \\ Neurosciences Medical University of \\ South Carolina, Charleston, SC, USA
}

Correspondence: Colleen A Hanlon Departments of Psychiatry and Neurosciences, Center for Biomedical Imaging, Medical University of South Carolina, Charleston, SC, USA

Tel +l 8437925732

Fax + I 8437927457

Email hanlon@musc.edu

\begin{abstract}
Within substance abuse, neuroimaging has experienced tremendous growth as both a research method and a clinical tool in the last decade. The application of functional imaging methods to cocaine dependent patients and individuals in treatment programs, has revealed that the effects of cocaine are not limited to dopamine-rich subcortical structures, but that the cortical projection areas are also disrupted in cocaine dependent patients. In this review, we will first describe several of the imaging methods that are actively being used to address functional and structural abnormalities in addiction. This will be followed by an overview of the cortical and subcortical brain regions that are most often cited as dysfunctional in cocaine users. We will also introduce functional connectivity analyses currently being used to investigate interactions between these cortical and subcortical areas in cocaine users and abstainers. Finally, this review will address recent research which demonstrates that alterations in the functional connectivity in cocaine users may be associated with structural pathology in these circuits, as demonstrated through diffusion tensor imaging. Through the use of these tools in both a basic science setting and as applied to treatment seeking individuals, we now have a greater understanding of the complex cortical and subcortical networks which contribute to the stages of initial craving, dependence, abstinence, and relapse. Although the ability to use neuroimaging to predict treatment response or identify vulnerable populations is still in its infancy, the next decade holds tremendous promise for using neuroimaging to tailor either behavioral or pharmacologic treatment interventions to the individual.
\end{abstract}

Keywords: addiction, neural circuit, functional MRI, diffusion, brain stimulation, connectivity

\section{Introduction and history}

While the US Congress designated the 1990s as the "Decade of The Brain", many of the initial advances were limited to traditional neurologic disease processes which had obvious structural pathology (as in acute and chronic stroke) or neurochemical aberrations (as in Parkinson's Disease). Within the past decade however, the application of novel neuroimaging methods to psychiatric diseases has substantially grown. As the field continues to advance, these techniques may ultimately expand our ability to identify vulnerability to drug use and potentially tailor treatment to the individual user.

Historically, substance dependence disorders, as with many psychiatric diseases, have not been characterized by obvious neurostructural or chemical pathology in a single region of the brain. Through a steadily increasing number of publications since the beginning of the 21 st century (Figure 1), however, we now know that the 


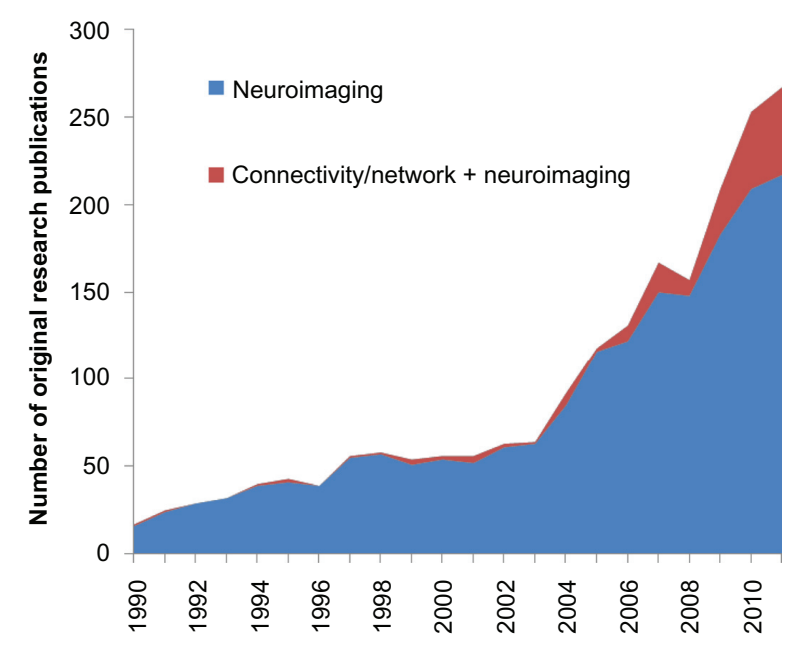

Figure I Number of original research publications using neuroimaging to study addicted populations users. The graph above demonstrates the results of a PubMed Central literature search for all original human research published between 1990 and $201 \mathrm{I}$ containing addiction related keywords* and either "neuroimaging" (blue) or connectivity-related (red) keywords".

Notes: *Addiction, substance dependence, cocaine, alcohol, marijuana, opiate, methamphetamine, nicotine; "neural circuit, connectivity, network.

continuum of substance use, dependence, abstinence, and relapse is associated with a cascade of dysregulation which likely begins in ventral areas of the striatum and expands to effect dorsal regions within the striatum as well as frontal and temporal cortical areas that project to the striatum.

While much of the original literature on the neural circuitry of addiction was done in rodent and non-human primate literature, powerful new functional connectivity measurements using magnetic resonance imaging in humans have allowed us to model these alterations in functional connectivity in cocaine users. Not only have these techniques provided us with the opportunity to translate the basic science research on acute and chronic cocaine use to clinical populations, they have also enabled us to investigate differences in individuals that are able to successfully abstain from cocaine from those that are not - a uniquely human and clinically critical aspect of addiction.

In this review, we will first describe several of the imaging methods that are actively being used to address functional and structural integrity in addiction. This will be followed by an overview of (1) the brain regions that are most often cited as dysfunctional in substance dependent individuals, with a specific emphasis on cocaine dependence, and (2) the growing research on functional and structural connectivity disruptions in these patients.

\section{Methods used to image}

Much of our understanding of the neurobiological consequences of chronic drug use in humans has come from imaging studies using positron emission tomography (PET) and functional magnetic resonance imaging (fMRI) to measure cerebral metabolism, blood flow, or blood volume. In this section we present some of the neuroimaging methods commonly used to study cocaine addiction along with promising new techniques. These methods have various strengths and weaknesses regarding their application to measuring brain structure and functional activity in addicts over time (Table 1).

\section{Structural magnetic resonance imaging (MRI)}

MRI is a powerful technique that uses the principles of nuclear magnetic resonance to create a high resolution image of different tissue types in the body. The resolution and quality of the tissue differentiation signal is associated with many aspects of the MRI protocol but one of the most apparent is the strength of the magnetic field. While most human imaging studies are now conducted in 3 Tesla MR scanners, animal imaging studies are now commonly conducted in 7 and 9 Tesla MR scanners. This higher field strength enables investigators to ask more precise questions about localization of function and integrity of tissue.

As a brief summary, the central principle of MRI is that through alignment of hydrogen atoms to a magnetic field, the structural composition of the tissue type can be determined by calculating the time that it takes for these atoms to return to their previous, unaligned state. Hydrogen atoms all have a magnetic spin and these spins are typically randomly distributed. In the presence of a magnetic field, however, these protons will align with a gradient induced by the MR scanner. Once aligned to a magnetic gradient, the atoms located in the numerous tissue types in the brain will drift back to their baseline state.

Although there are many different MR sequences (each designed to maximize contrast on a specific tissue type), the T1 contrast is among the most frequently acquired in imaging studies of addiction. In a brain image that was acquired with T1-weighted contrast, the gray matter of the cortex and the subcortical nuclei appear dark, the myelin of the axonal pathways is lighter in color, and the cerebrospinal fluid in the ventricles is black. This grayscale separation allows for analysis of the brain structure of cocaine users.

A common method for quantitatively measuring structural differences among cocaine users is voxel-based morphometry (VBM). Briefly, through segmentation of the T1 image into three component parts (gray matter, white matter, and cerebrospinal fluid), and spatial transformation of these components to a common anatomical template, it is 


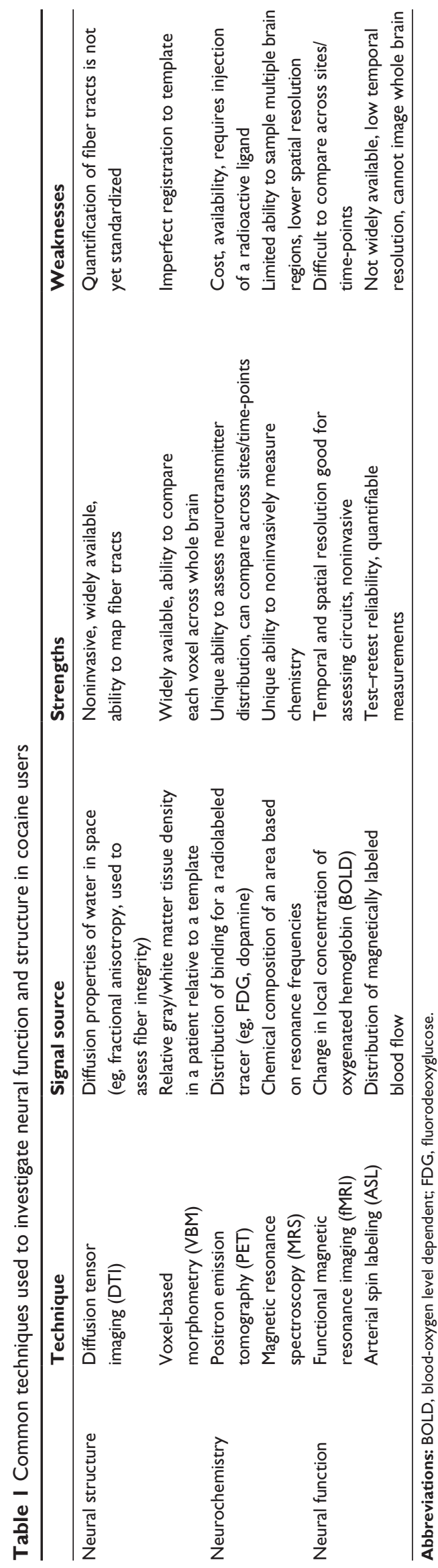

possible to infer region specific changes in brain atrophy or enlargement. There are several studies which have used VBM to characterize alterations in tissue density among cocaine users and abstainers. ${ }^{1-3}$

\section{Diffusion tensor imaging (DTI)}

Another powerful anatomical imaging technique using magnetic resonance technology is diffusion tensor imaging. Whereas typical gradient echo imaging for structural MRI provides high resolution data regarding the composition of the brain at a specific location, DTI employs a series of gradients to generate information about diffusion within the brain. ${ }^{4}$ Although DTI has the ability to assess many different tissue types, the most common application of this technique in addiction literature is to quantify the integrity of axon tracts ("white matter") in the brain. This is accomplished by calculating the Brownian motion of water molecules following the application of magnetic gradients from multiple directions.

Anisotropy is a term used to express that something has directional properties, rather than being a perfect sphere. Fractional anisotropy (FA) is a commonly used metric to quantify the results of DTI studies. Areas with high diffusion coefficients (eg, the lateral ventricles in which the diffusion of water molecules are not encumbered by tissue structure) have low anisotropy. Areas with low diffusion coefficients (eg, the corpus callosum in which the diffusion of water molecules is restricted by the bundles of axons) have high anisotropy. Consequently, DTI provides insight into the integrity of long range axonal fiber tracts connecting different areas of the brain. Among cocaine users, DTI has revealed that white matter integrity in the frontal lobe is decreased among cocaine abusers. ${ }^{5-7}$ Moreover, the decrements in the integrity of the frontal lobe have been associated with greater impulsivity among users, ${ }^{6}$ and shorter length of abstinence among a group of cocaine users receiving treatment. ${ }^{7}$

\section{Magnetic resonance spectroscopy (MRS)}

A relatively new method being used to study cocaine and the brain, and offering a way to examine the biochemical composition of the brain noninvasively is MRS. ${ }^{9}$ Rather than examine the structural integrity or tissue composition in the brain, spectroscopy assesses the chemical composition of neural tissue. Using the same principles of nuclear magnetic resonance that create the standard anatomical MR contrasts, spectroscopy calculates the contribution of a suite of protein metabolites (each with its own nuclear magnetic resonance frequency) that are present in the neural tissue. 
Although the suite of metabolites that can be identified has been relatively limited to date (creatine, lactate, myoinositol, $\mathrm{N}$-acetylaspartate), increasingly sophisticated imaging techniques such as chemical shift imaging are being developed to differentiate between various neurotransmitters (such as glutamate and $\gamma$-aminobutyric acid) which has historically been difficult due to the similarity in their resonance signatures.

Recent papers using MRS showed lower levels of glutamate in the rostral anterior cingulate cortex (ACC; associated with cognitive control) in cocaine users versus controls and that glutamate levels in the dorsal ACC (associated with reward response) were positively associated with impulsivity among users. ${ }^{11,12}$ Further, Schmaal and colleagues demonstrated that $\mathrm{N}$-acetylcysteine, which has been studied for treating cocaine addiction, ${ }^{13}$ acutely decreased glutamate levels in the dorsal ACC among cocaine users. ${ }^{11}$ MRS has also demonstrated decreased levels of $\mathrm{N}$-acetylaspartate, a neuronal marker, in the frontal lobes of cocaine users, indicating neuronal injury. ${ }^{14}$

\section{Functional magnetic resonance imaging (fMRI)}

Whereas structural MRI and DTI provide information about the composition of the brain at a single time-point, fMRI is a very common imaging approach that provides information about the functional activity of the brain over time. This is achieved through the assessment of oxygenated and deoxygenated hemoglobin, each of which can be differentiated via their magnetic properties. Information in the brain is transmitted when populations of neurons send action potentials to their synaptic terminals, which release various neurotransmitters that in turn bind with postsynaptic receptors on neighboring neurons. This neural activity requires oxygen, which is delivered to the site of activity via hemoglobin molecules in the blood. In fMRI studies, neural activity is approximated by assessing the alterations in the ratio of oxygenated to deoxygenated blood in a specific brain region. This is referred to as blood-oxygen level dependent (BOLD) activity, and is frequently described as the BOLD signal.

Functional MRI is typically used to investigate regional brain function by having participants engage in tasks while they are in the MR scanner. For example, participants may be asked to view cocaine-related versus neutral pictures or to complete a motor task by mimicking finger movements with a response box. The time course of BOLD signal throughout the experimental conditions is then analyzed both within and between participant groups to determine which brain regions were more active during those tasks relative to a control. The flexibility of functional MRI has enabled it to be applied to addiction in many different ways.
Within addiction literature, fMRI has been widely applied to address questions that range from the acute effects of cocaine on the brain to uncovering patterns of neural activity that predict successful treatment outcomes. Studies using fMRI have found that when cocaine users are exposed to cocaine cues (eg, images, video), cortical and limbic regions associated with reward and motivation, memory, and learning (eg, ACC, medial and inferior parts of the prefrontal cortex, caudate) have increased BOLD signal, supporting the link between cocaine use and dopaminergic pathways. ${ }^{15-17}$

\section{Arterial spin labeling (ASL)/cerebral blood flow measurement}

ASL is a noninvasive functional imaging method which uses the same properties of MR mentioned in the previous techniques to label water in the arterial blood flowing into the brain region (or slice) of interest. The magnetically labeled water in the arterial blood is essentially treated as a noninvasive tracer as it enters brain tissue. ${ }^{18}$ As opposed to BOLD imaging, in which the intensity of activation in a brain region during a specific task or event can only be interpreted relative to another task, event, or brain region, the ASL technique provides an absolute measurement of the amount of blood that was delivered to the brain region of interest (ROI).

The contrast in ASL is achieved by first magnetically labeling the arterial blood below a selected ROI and, as it enters the ROI, acquiring this magnetically tagged image. A subsequent control image is then taken without the labeled arterial blood, and subtracted from the tagged image, creating a perfusion image, allowing for a quantification of cerebral blood flow (CBF) into the region. This multi-step process can take more than twice as long as the acquisition of a BOLD image. A potential benefit of ASL over BOLD imaging, however, is the lower variability between subjects - which could allow for smaller sample sizes to detect effects - and greater reliability of measurement within subjects over scans. ${ }^{19,20}$ ASL is a relatively new method being used to study addiction, and there are no currently published studies using this approach to investigate cocaine abuse among humans. ASL is however being used to study other addictive processes, such as alcohol abuse, and to longitudinally assess changes during abstinence or relapse. ${ }^{21,22}$ ASL shows promise as a new tool for elucidating the mechanisms of cocaine abuse.

\section{Positron emission tomography (PET)}

Whereas the previous imaging techniques all use the principles of nuclear MR to generate images of neural structure and 
function, PET is an extremely powerful neuroimaging technique which can quantify the binding of various neurotransmitters in the brain (such as dopamine or serotonin) as well as monitor functional activity via glucose consumption. This image is generated after the injection of safe amounts of radioactive tracers, which contain positron-emitting isotopes that bind to specific elements of interest in the brain (eg, glucose, dopamine). The PET scanner generates a three-dimensional image of the brain which includes the location and density of the tracers at their binding sites, indicating areas of increased activity (eg, glucose metabolism, CBF).

Although PET experiments typically result in a single image of radioligand binding and thereby lack the temporal resolution of $\mathrm{fMRI}$, the ability to label the spatial distribution of specific neurotransmitters, including dopamine receptors and glucose metabolism, has had significant impact on our knowledge of cocaine's effects on the brain..$^{23,24}$ Over the past two decades, Volkow and colleagues have published a large repertoire of publications on alterations in glucose metabolism and dopamine binding density in cocaine users. Among the most influential of these publications is the identification of compromised dopamine systems and lower metabolic rate of glucose in both frontal and limbic regions in current and abstinent cocaine users, a phenomenon now referred to as hypofrontality. ${ }^{8}$

\section{Neural regions involved in addiction}

The multiple neuroimaging methods used to study the relationship between cocaine and the brain have consistently found alterations in both cortical and subcortical brain regions. These regions are important in cocaine abuse, including the emotional response to drug cues, or craving, and the controlled regulation of those responses. With advancements in imaging and analytical methods, over time we have learned not only of the areas important in cocaine abuse, but how they connect and work as a functional network. Information about both morphology and BOLD signal in different brain regions continues to provide a clearer image of cocaine addiction in the brain. In this section, we describe some of the regions repeatedly found to be important in cocaine abuse (Figure 2).

\section{Subcortical regions}

The primary site of action of cocaine is in the dopaminerich regions of the ventral striatum, including the nucleus accumbens (NAcc) and the ventral tegmental area (VTA). Although it is relatively scarce in the brain relative to glutamate and gamma-Aminobutyric acid, dopamine is the

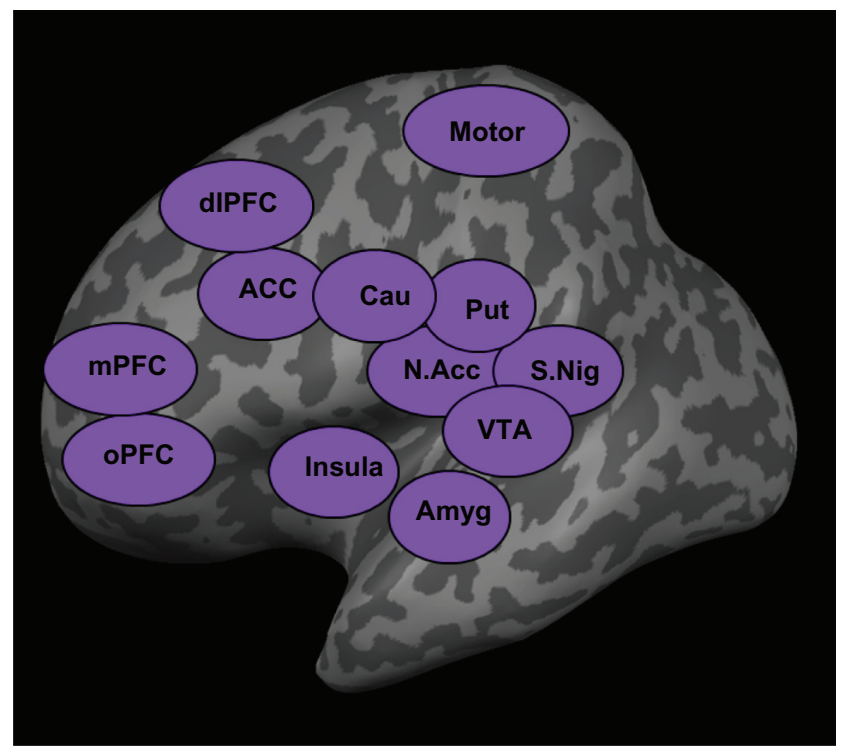

Figure 2 Cortical and subcortical brain areas frequently cited in cocaine addiction literature. Brain regions which are frequently investigated in both clinical and preclinical cocaine neuroimaging studies: orbital prefrontal cortex (OPFC), medial prefrontal cortex (MPFC), anterior cingulate cortex (ACC), insula, caudate (Cau), putamen (Put), ventral tegmental area (VTA), nucleus accumbens (N.Acc), amygdala (Amyg). The substantia nigra is not frequently assessed in cocaine literature but

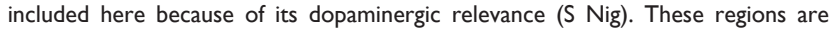
highly interconnected with some level of functional and structural organization.

primary neurotransmitter associated with governance of reward-related experience and learning. The primary mechanism of action of cocaine is to block reuptake of dopamine, thereby flooding the synapse with dopamine and leading to the euphoric and rewarding aspects of cocaine use. ${ }^{25}$ In proportion to the total brain volume of a typical adult, the NAcc and the VTA are very small (approximately $<1 \mathrm{~cm}^{3}$ on the standardized Montreal Neurologic Institute template compared to the brain which is over $1100 \mathrm{~cm}^{3}$ ). While this poses a challenge for human functional imaging techniques which usually have a maximal resolution of $6 \mathrm{~mm}$ for PET and $2 \mathrm{~mm}$ for BOLD, several high resolution imaging studies have been able to assess alterations in these regions in cocaine users.

\section{Ventral tegmental area (VTA)}

The VTA is a structure in the midbrain where the dopaminergic projections to limbic and cortical areas originate, making it a key component of the reward circuitry implicated in addiction. The VTA is a small structure and difficult to resolve in whole brain imaging of humans due to size and partial volume effects. Consequently, activity related to this region is often not reported in studies on cocaine. Acute cocaine administration in the MR scanner, has been associated with elevated activity in the VTA. This elevated BOLD signal in the VTA has been correlated with the "rush" associated with 
cocaine. ${ }^{24}$ Another study however, demonstrated lower BOLD signal in the VTA following administration. ${ }^{27}$

\section{Nucleus accumbens (NAcc)}

The NAcc receives afferent projections from the VTA. It is dopamine rich and the increase in dopamine levels in the NAcc produces the positive, rewarding affective response of cocaine. Both fMRI and PET studies have demonstrated altered NAcc activation during cue-elicited craving, selfreported craving, and during cocaine administration. ${ }^{15,26-28}$ Following acute administration of cocaine in the MR scanner, activity in the NAcc was negatively correlated with reports of cocaine high and positively correlated with craving. ${ }^{26,29}$ Thus, activity in this component of reward circuitry of the brain may be suppressed with the acute effects of cocaine, but increased when the craving reinstates.

\section{Dorsal striatum (or basal ganglia)}

As individuals transition from casual to habitual cocaine use, there is likely an expansion of dysfunction from the ventral striatum (linked with motivation and reward) to the dorsal striatum (reflecting habitual behavior). This transition has been elegantly demonstrated in rodent ${ }^{30}$ and non-human primate work. ${ }^{31}$ Although longitudinal studies that could capture this transition are difficult to do in humans, studies have demonstrated that while acute cocaine use is mediated by the ventral striatum, ${ }^{29}$ after chronic cocaine use (12 or more years), these substance-dependent individuals have dysfunction throughout their dorsal striatum. ${ }^{32}$ The dorsal caudate and the putamen form the dorsal striatum. Strongly connected with the cortical targets of the mesocortical dopamine systems, the dorsal striatum is involved in decision making, reward-related learning, movement, and the processing of sensorimotor, cognitive, and emotional information. ${ }^{33}$

\section{Caudate nucleus}

The caudate is generally involved with goal-directed and motivated behavior ${ }^{33}$ and is active during cue-elicited craving. ${ }^{15,28}$ Increased BOLD signal in the caudate has been found when users report feeling the rush and high from cocaine administration. ${ }^{26,29}$ The volume of the caudate may be larger in cocaine users than controls, ${ }^{2}$ which has been further related to impaired attention. ${ }^{34}$ In a study with patients in treatment for cocaine abuse, Sinha and colleagues found that when cocaine users were instructed to imagine stressful situations while in the scanner, they had increased BOLD signal in the caudate. Activation in the caudate during this stress task was also associated with an increase in cocaine craving. ${ }^{35}$

\section{Putamen}

The putamen is generally involved in movement and learning ${ }^{33}$ and its volume is larger among cocaine users. ${ }^{34}$ During a working memory task for example, cocaine users have lower activity than healthy controls in the putamen, an area critical for successful performance. ${ }^{37}$ Furthermore, in an innovative treatment study, Brewer and colleagues determined that lower activity in the putamen during a Stroop color-naming task prior to enrolling in a drug treatment program, predicted a shorter length of abstinence in treatment-seeking cocaine users. ${ }^{38}$ Although typically assigned to the sensorimotor and cognitive aspects of cocaine addiction, activity in the putamen is also correlated with levels of craving following cocaine administration. ${ }^{26}$ Using PET, Wong and colleagues demonstrated elevated levels of dopamine receptor occupancy in the putamen when cocaine users were experiencing cue-induced craving. ${ }^{36}$

\section{Thalamus}

Likely the largest hub of afferent and efferent connections in the brain, the thalamus is actually a complex of four nuclei which act as relays between cortical and subcortical structures. Among cocaine-dependent individuals, the volume of gray matter in the left thalamus is lower than in controls. ${ }^{39}$ Compared to controls, cocaine users have decreased BOLD signal in the thalamus during visual attention and memory tasks. ${ }^{37,40}$ Using fMRI, deactivation in the thalamus during a working memory task has been associated with less effectiveness of treatment as measured by urine screens. ${ }^{35}$ BOLD activity in the thalamus has been associated with the high from acute administration ${ }^{26,29}$ and cocaine cue exposure. ${ }^{15}$

\section{Amygdala}

The amygdala is a subcortical structure which receives inputs from the thalamus and hippocampus, and is important in the interpretation of the salience of sensory information and reinforcement. ${ }^{41}$ The amygdala directs attention and emotional response, memory formation, and instrumental behavior. For instance, increased amygdala activation in response to implicit cocaine cues correlated with positive affective response ratings to the cues - demonstrating its importance in the identification of the salience of drug-related stimuli even outside of awareness. ${ }^{42}$ Amygdala volume is smaller among cocaine-dependent individuals relative to controls ${ }^{43}$ and activation in the amygdala is increased during cueelicited craving. ${ }^{27,44-46}$ Using PET, Childress and colleagues found increases in $\mathrm{CBF}$ in both the amygdala and $\mathrm{ACC}$ in cocaine users versus controls while viewing cocaine-related videos..$^{45}$ 


\section{Cortical regions}

In addition to functional and neurochemical alterations in the dopamine-rich subcortical areas, chronic and acute exposure to cocaine is associated with functional and structural irregularities in cortical areas that project to these subcortical domains. One consistent finding in neuroimaging studies is that of "hypofrontality", or decreased function of the prefrontal cortex of cocaine abusers when compared with non-drug using controls. This dysfunction in the prefrontal cortex has been characterized by profound changes in glucose metabolism, dopamine density, BOLD signal, and neural tissue density associated with cocaine abuse.

\section{Prefrontal cortex (PFC)}

The PFC occupies the frontal section of the brain, anterior to the precentral gyrus, and is involved in many higher order processes such as planning, decision making, inhibition, coordinating purposeful behavior, and top-down cognitive processes which are controlled and effortful. ${ }^{47}$ Areas of the PFC are connected to limbic regions and are involved in emotional processing as well. Major segments of the PFC important in addiction include the medial PFC (mPFC, Broadmann Area, BA 9), the dorsolateral PFC (DLPFC, BA 46), and the OFC (BAs 10, 11, 47).

\section{Medial prefrontal cortex (mPFC)}

The mPFC extends throughout the medial section of the frontal cortex and into the OFC. Studies using PET and fMRI have shown that cocaine users have increases in $\mathrm{mPFC}$ activity when exposed to cocaine versus neutral cues. . $^{15,44,48}$ Using VBM, researchers have shown that gray matter volume in the mPFC reduced compared to controls. ${ }^{2,49}$ The mPFC also plays an important role in the cognitive decrements seen in cocaine users. Functional imaging studies have shown general hyopactivation in the mPFC among users, relative to controls, during cognitive and attention tasks. ${ }^{50-52}$ Moreover, hypoactivation of the ventral $\mathrm{mPFC}$ during a cognitive task (Stroop) in cocaine users prior to entering treatment has also predicted shortened length of abstinence..$^{38}$

\section{Dorsolateral prefrontal cortex (DLPFC)}

The DLPFC sends afferent projections to the dorsal striatum, and is involved in higher order processes, such as conscious decision making, reasoning, working memory, inhibition, as well as outcome prediction. ${ }^{53}$ Cocaine users have a significant reduction in cortical thickness in the DLPFC compared to controls. ${ }^{52}$ The DLPFC is active during craving ${ }^{44}$ and PET and fMRI studies have shown increased activation when cocaine users are presented with drug-related stimuli and given cocaine injections. ${ }^{16,27,44,55}$ Abstinent cocaine users have significantly less activity in the DLPFC as quantified via PET when engaging in decision-making tasks involving reward compared to nonusers. ${ }^{52}$ However, among active cocaine users, less BOLD signal in the DLPFC during a cognitive task (Stroop) prior to entering treatment predicted longer stays in treatment. ${ }^{38}$ Considered together, these data suggest that users with more efficient use of the DLPFC may do better in treatment, but these levels may not be restored to those of non-drug using controls following abstinence.

\section{Orbitofrontal cortex (OFC)}

The OFC is located on the ventral surface of the prefrontal cortex, is part of the mesolimbic dopamine system and contributes to reinforcement of cocaine (eg, with NAcc, thalamus, ventral striatum, and indirectly with amygdala, cingulate, hippocampus). Given its strong connectivity to limbic regions as well as the MPFC and ACC, the OFC likely integrates the reinforcing effects of cocaine taking with the attentional aspects of cocaine seeking. ${ }^{56}$ The OFC has been implicated in response inhibition and its dysregulation and may contribute to the continued use of cocaine after the rewarding effects no longer are experienced. Thus, disruption of the OFC has been found to be related to risky decision making and an inability to anticipate outcomes..$^{53}$ It has also been associated with perceiving the value of a stimulus, such as reward, and learning the association between a stimulus and outcome. ${ }^{57}$

As with other frontal areas, the volume of gray matter in OFC is lower among cocaine-dependent individuals than nondrug using controls ${ }^{49}$ and this decreased volume has been linked with greater cocaine-related compulsivity and longer duration of use. ${ }^{1,2,39}$ Functional neuroimaging has shown that the OFC is active during self-reported craving, and has been negatively correlated with reports of feeling high after administration. ${ }^{29,44}$ The OFC is active in response to both implicit and explicit cocaine cues. ${ }^{42,44}$ Activation in both the OFC and DLPFC has been repeatedly linked with drug cue exposure among cocaine users, but not with cocaine abstainers (or those in treatment).$^{58}$ This may reflect differences in the ability to exert control over drug-seeking behaviors of those able to abstain. Furthermore, recent data from Volkow and colleagues ${ }^{59}$ suggest that individuals that are able to successfully lower their craving in the presence of cues, have a corresponding decrease in activity in the OFC, an insight which may be valuable in future treatment initiatives. 


\section{Anterior cingulate cortex (ACC)}

The ACC is part of the limbic system and is involved in the regulation of attention and emotion, inhibitory control, error monitoring, and motivation. ${ }^{60}$ Dysregulation of the ACC in cocaine users may underlie their inability to control their cravings. Cocaine users have decreased gray matter density in the ACC compared to controls, ${ }^{49}$ and this reduction has been found to be greater for those with a longer history of abuse. ${ }^{1,2}$ In addition, numerous reports have demonstrated that cocaine users have lower rates of glucose usage as measured with fluorodeoxyglucose (FDG) and PET, particularly in frontal regions including the cingulate and OFC. ${ }^{61-65}$ These depressed rates of functional activity have been reported to persist for up to 3 months of abstinence. ${ }^{8}$

Following cocaine administration there is an elevation of BOLD signal in the $\mathrm{ACC}^{15,16,29}$ which has been correlated with aspects of the cocaine high. ${ }^{26,29}$ Cocaine users have decreased BOLD signal relative to controls in the ACC when completing a task requiring cognitive inhibition ${ }^{50,66}$ and visual attention. ${ }^{51}$

\section{Insula}

The insula is a cortical structure located deep in the brain between the frontal and the temporal lobes, largely divided into the anterior and posterior insula. It has structural connections with many of the areas involved in addiction, including the amygdala, basal ganglia, thalamus, $\mathrm{OFC}$, and $\mathrm{PFC}$, giving it an important role in the neural circuitry of cocaine abuse. ${ }^{67}$ The insula is involved in emotion processing and arousal including awareness of one's own bodily states, as well as decision-making and other executive processes. Increased activation in the insula has been found when users experience cocaine craving and high as measured by PET and fMRI. ${ }^{27,44,46}$ Insula volume is reduced among cocaine users, and longer use of cocaine is correlated to smaller insula volume. ${ }^{2}$ In addition, smaller insula volume among cocaine users and abstainers is associated with decrements in attentional control. ${ }^{2,3}$

\section{From regions to circuits}

In addition to learning about activated regions, researchers are interested in understanding how these brain regions relate to one another, or their level of connectivity. The brain is a dynamic system with many areas activated simultaneously at different intensities and responsive to different aspects of the environment. Nearly all behaviors in the human repertoire are the result of a complex interaction requiring efficient electrochemical transmission between cortical and subcortical brain regions.
As displayed in Figure 3, pyramidal neurons in many of the cortical areas discussed above, project to subcortical targets via long, myelinated axons. Specifically, when the membrane potential of the cortical neuron (summated by the dendrites and soma) are sufficient to produce an action potential, an electrical signal propagates down the axon towards the subcortical target. The axon terminates at the synapse, where neurotransmitters will be released and affect the membrane potential of the subcortical neuron. The axon is covered in a myelin sheath which is required to maintain the electrical signal integrity. The myelin is formed by glial cells which are bright on a structural (T1 image) MRI ("white matter"), while the dendrites and cell bodies of the neurons are darker on a T1 image ("gray matter").

Connectivity analysis allows researchers to look at networks or circuits of brain regions to provide insight into how the brain regions work together to produce functional changes. Connectivity analysis occurs in two forms called functional and effective. ${ }^{68}$ Functional connectivity examines temporal correlations of spatially remote, neurophysiological events, or rather how different regions correlate in time. Effective connectivity examines the causal influence of one brain region on another.

\section{Frontostriatal dopamine circuits}

While many neural circuits have been implicated in the behavioral manifestations of substance abuse, dependence, and relapse, the frontostriatal dopamine circuits are among the most frequently investigated. The mesocortical (associated with attention/executive processing) and mesolimbic (associated with reward/affective processing) dopamine circuits are thought to govern the alterations in cognitive control, impulsivity, and habit formation experienced by

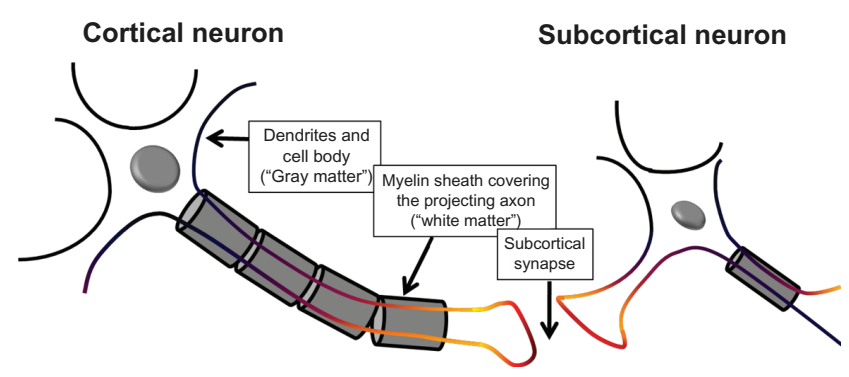

Figure 3 A representative cortical neuron projecting to a subcortical neuron. Information in the brain is processed via neural circuits like these which rely on both intact functioning of the neurons and the structural integrity of the axons and myelin which will transmit the electrical potential. Both functional and structural connectivity of cortical-subcortical circuits appear to be compromised in chronic cocaine users.

Notes: This figure of two pyramidal neurons is for display purposes only. It is not to scale. There are also reciprocal subcortical-cortical projections. 
cocaine users. ${ }^{62}$ Animal studies have demonstrated that the mesolimbic dopamine system mediates the rewarding, reinforcing properties of cocaine. As drug use progresses to abuse, the rewarding properties of the drug become less salient, and drug taking becomes guided by conditioned responses and habit formation, which are mediated by the mesocortical system. ${ }^{30,31}$

\section{Imaging circuits in substance abuse populations}

The functional and effective connectivity of neural circuits can be measured in several ways: (1) during an active state in which participants are engaged in a task, (2) during a resting state in which participants are not actively engaged in a task, and (3) during a hybrid situation in which external brain stimulation is used to activate neural circuits while a participant is resting.

\section{Functional connectivity during task performance}

A recent study by our laboratory group used a region-based functional connectivity analysis to address alterations in sensorimotor function in cocaine-dependent individuals. A prior study in this population had demonstrated that cocaine users have elevated activity in several cortical motor regions during a simple sensorimotor integration task. Although the cocaine users had significantly longer reaction times and made more errors than the controls, the BOLD signal in these cortical regions did not correlate with the behavioral impairment. ${ }^{69}$

Consequently, we shifted our focus from looking at individual brain regions in isolation to the functional interaction between the regions. Using a ROI approach, we extracted the time course from several brain regions which were involved in successful task performance and measured the degree of functional coupling (or connectivity) via cross correlation in the cocaine users and healthy controls. These data revealed cocaine users had a selective impairment in coupling within the cortical-striatal connections. Cortical-cortical coupling however, was intact (Figure 4). ${ }^{69}$ The specificity of this deficit to frontostriatal connections suggests that cocaine addiction may share common neurobiological mechanisms with other clinical conditions such as obsessive compulsive disorder, ${ }^{71}$ Tourettes syndrome, ${ }^{72}$ and attention deficit disorder. ${ }^{73}$

\section{Functional connectivity during resting state}

Another way researchers are investigating the brain of cocaine users is by looking at networks of activations during a resting state. In resting state studies, participants lie in the scanner awake, instructed to keep their brain at rest typically for 6 minutes or more. These studies have found specific brain circuits to be active during the absence of stimulation, or while at rest. Resting state studies of addicted individuals have found alterations in the normal resting state circuitry. ${ }^{74-76}$ For instance, connectivity, or correlated activity, between regions known to be important in cocaine addiction (eg, VTA, ACC, amygdala, thalamus) was decreased at resting state in cocaine users relative to controls, and less connectivity strength among the VTA to the thalamus and NAcc was correlated with more years of cocaine use. ${ }^{74} \mathrm{Li}$ et al demonstrated that, at rest, acute cocaine administration decreases the functional correlation of voxels within isolated brain regions. ${ }^{77}$ These findings suggest that disruption of the reward system in cocaine users can be detected even in the absence of relevant cues.

\section{Functional connectivity assessed via brain stimulation}

While much of the focus of brain imaging studies to date has been "mapping" the neural circuits involved in various components of addiction, there is an emerging interest in "modulating" those neural circuits through brain stimulation techniques such as transcranial magnetic stimulation (TMS). With TMS, a coil that generates a magnetic field is placed over the scalp and produces electrical changes in the brain. The frequency at which the currents are generated can result in inhibition or excitation of activity in brain regions beneath the coil. Thus TMS can be used to probe specific regions and temporarily alter brain activity,

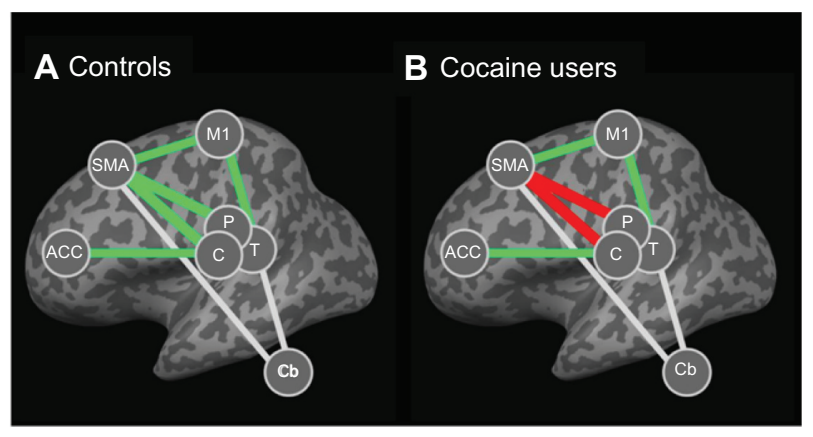

Figure 4 Cortical-striatal connectivity deficits during a simple sensorimotor integration task in cocaine users. Reprinted from Drug and Alcohol Dependence. Hanlon CA, Wesley MJ, Stapleton JR, Laurienti PJ, Porrino LJ. The association between frontal-striatal connectivity and sensorimotor control in cocaine users. Drug Alcohol Depend. 201 I; I 5(3):240-243. Copyright 201 I, with permission from Elsevier. ${ }^{70}(\mathbf{A})$ During a simple finger tapping task (which requires frontal and striatal coordination including the dopamine system) controls have a significant functional coupling between both cortical-cortical regions and cortical-subcortical regions. (B) In cocaine users however, cortical-subcortical coupling was impaired despite intact cortical-cortical coupling. 
providing insight into causal processes of addiction. It is now possible to investigate alterations in functional and structural connectivity in chronic cocaine users and abstainers via simultaneous brain imaging and brain stimulation. This technique, often referred to as interleaved TMS/BOLD imaging ${ }^{78,79}$ provides us with a unique opportunity to probe neural circuitry.

A recent study from our laboratory used an optimized interleaved TMS/BOLD sequence in two cortical targets to examine the differential activation of lateral (or mesocortical) and medial (or mesolimbic) neural circuits, which are known to be important in drug abuse. Interleaved TMS/ BOLD imaging data was acquired for ten healthy individuals who received TMS in two runs with the coil positioned over the: (1) DLPFC and (2) mPFC. We found that DLPFC TMS was associated with a significant elevation of BOLD signal in multiple dorsal cortical areas, whereas mPFC TMS was associated with a significant elevation of BOLD signal in multiple medial and limbic subcortical areas (Figure 5). These preliminary data demonstrate that it is possible to differentially activate known cortical-subcortical networks through brain stimulation and has important implications for both basic neuroscience research and in patient populations with pathology differentially affecting mesolimbic versus mesocortical circuitry.

\section{Structural connectivity: white matter}

As described above, the last 15 years have been a period of tremendous growth in our knowledge of the cortical and subcortical brain regions affected in cocaine users and abstainers. Furthermore, there is a growing body of research on alterations in functional connectivity in cortical-striatal circuits of cocaine users. While pathology in these neural circuits may be related to compromised function of the neural gray matter (eg, cell loss, dendritic spine growth, receptor density changes), it may also be related to compromised structure in the fiber tracts which connect one region of the circuit to the next ("white matter").

Structural abnormalities of both gray matter and white matter in cocaine users are well established. ${ }^{5,80-84}$ These studies consistently report that the volume and tissue density of the PFC is smaller in cocaine users than nondrug using controls, and may be related to length of use. ${ }^{84}$ Bartzokis and colleagues investigated white matter volume in a large cohort of cocaine-dependent individuals and demonstrated that cocaine-dependent individuals did not have the same age-related increases in white matter volume observed in non-drug using controls, suggesting an arrested development of white matter. ${ }^{85}$ While several studies have demonstrated that after several weeks of abstinence, former cocaine users still have lower PFC gray matter, ${ }^{49,81}$ individuals that are able to remain abstinent for at least 30 days have no difference in prefrontal gray or white matter relative to non-drug using controls. ${ }^{3}$

The development of DTI has complemented the rise of functional connectivity as it provides insight into the structural basis for impaired functional connections. One of the primary outcome measures of DTI studies is fractional anisotropy (see "Methods used to image" section) which is

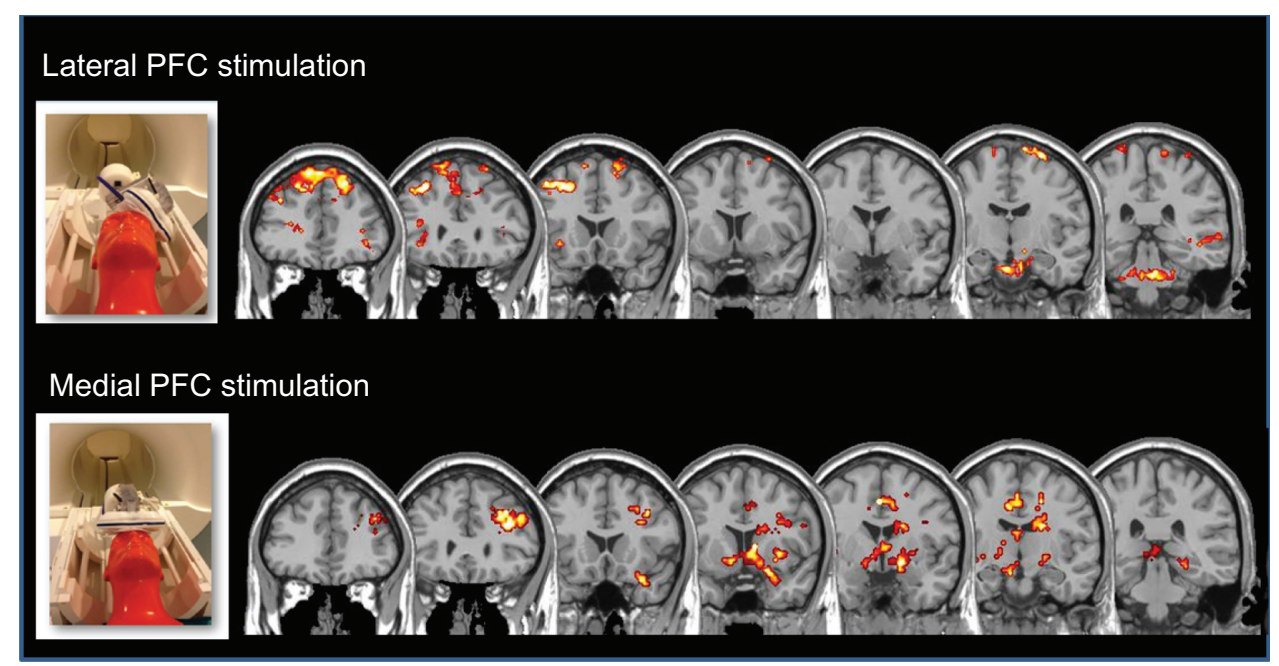

Figure 5 Measuring functional connectivity via simultaneous brain imaging and brain stimulation. Through the use of transcranial magnetic stimulation in the magnetic resonance environment, we are able to selectively stimulate cortical brain regions and measure the BOLD response or changes in blood flow secondary to the stimulation. Abbreviation: BOLD, blood-oxygen level dependent. 
an indirect measure of the degree of directional diffusion in that brain area. Specifically, brain areas in which water must diffuse along an axon will have a higher FA value than brain areas without any barriers to movement (eg, cerebrospinal fluid).

One of the first studies to document alterations in the structural integrity of white matter fiber tracts in cocaine users was by Moeller et al. ${ }^{83}$ Using DTI, they demonstrated that cocaine-dependent individuals have lower FA values in the genu and body of the corpus callosum, the largest white matter tract of the brain which serves to integrate information between hemispheres. These authors also found a significant correlation between elevated impulsivity scores and lower FA values in the anterior segments of the corpus callosum in these cocaine-dependent individuals. The compromised structural integrity of callosal fibers is likely due to a loss in myelin ${ }^{86}$ and the association with high impulsivity has been subsequently replicated. .,6,87 $^{-1}$

Although there are no studies available to date integrating alterations in functional connectivity with changes in structural integrity of myelinated axons projecting between cortical and subcortical regions, this is likely to change within the next few years. Several groups are currently developing quantitative methods to unite functional and structural pathology. As demonstrated in Figure 6, in a recent functional imaging study by our group, a cohort of cocaine-dependent individuals and age- and gender-matched controls performed a simple right hand finger tapping task in an MRI scanner during BOLD image acquisition. ${ }^{69}$ During this task, in which controls use predominantly their left hemisphere, cocaine users have significantly greater activity in both the left and right primary motor cortex. DTI data from these individuals demonstrate that the users have significantly lower FA in the body of the corpus callosum (Figure 6A), and that the areas of lower FA values in the cocaine users are spatially coincident with areas of elevated functional activity (Figure 6B), suggesting the loss of the typically lateralized neural circuit is related to a loss of inhibitory transmission through the corpus callosum. As the sophistication and availability of tools allowing investigators to integrate multiple modes of neuroimaging data continues to grow, it is likely that the next decade of research in neuroimaging of neural circuits in addiction will shift towards uniting our knowledge of functional connectivity and structural integrity.

\section{Conclusion}

Cocaine abuse is a complex disorder. In the last 30 years, we have seen tremendous growth in imaging. As the technology continues to grow rapidly, the developments in our knowledge of this disease process also seem to grow. These methods have allowed us to see that the effects of cocaine are not limited to the VTA and NAcc. Instead, in humans, the effects of cocaine involve a complex network of cortical and subcortical circuits. As addiction progresses (and in abstinence), this network is also dynamic. Furthermore, recent work has demonstrated that these dynamic changes in functional connectivity are associated with alterations in structural integrity. With each year, technological and analytical developments, as well as devoted and creative researchers, are bringing us closer to an integrated understanding of the functional and structural alterations that occur in the brains of chronic cocaine users as well as the hallmarks of successful abstainers.

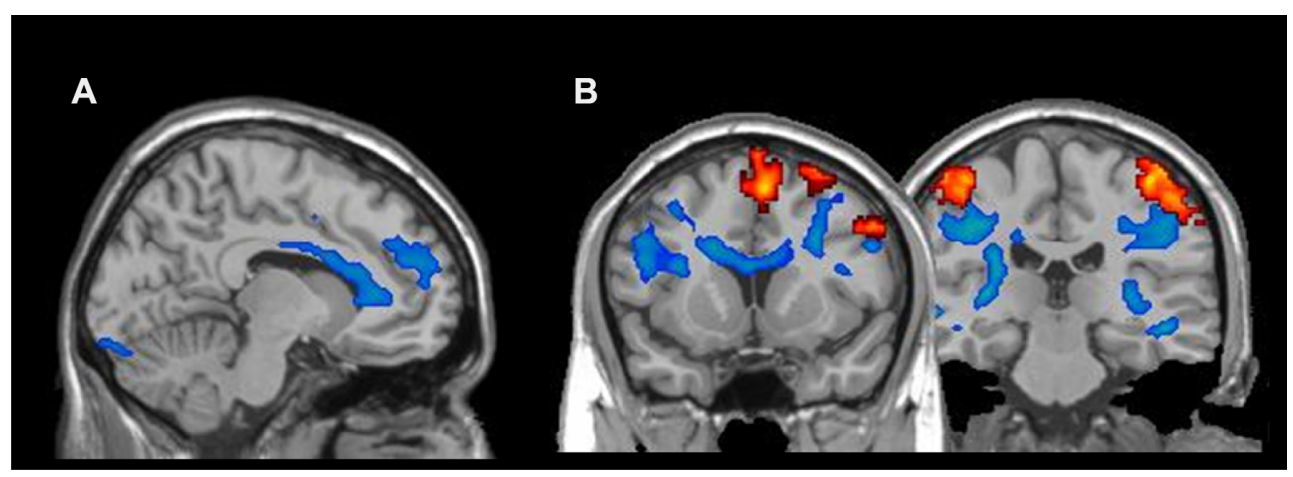

Figure 6 Integrating impaired structural integrity in cocaine users with functional activity. (A) Cocaine users ( $\mathrm{n}=26)$ have lower structural integrity of transcallosal fibers than controls $(\mathrm{n}=36)$ (fractional anisotropy values, $P<0.00 \mathrm{I})$. (B) Loss of structural integrity (blue) overlain with abnormal functional activity (orange) in chronic cocaine users during a basic finger tapping task.

Notes: As described in Hanlon et al, ${ }^{70}$ cocaine users have a loss of typical functional laterality during a simple finger tapping task. These data demonstrate that cortical areas of abnormally elevated BOLD signal are coincident with areas of decreased corpus callosum fiber integrity (blue). This suggests that the loss of typical laterality may be due to a loss of typical transcallosal inhibition via corpus callosal fibers.

Abbreviation: BOLD, blood-oxygen level dependent. 


\section{Disclosure}

This work was supported in part by National Institute of Drug Abuse grants K01DA0267756 (CH) and T32DA007288. The authors report no conflicts of interest in this work.

\section{References}

1. Franklin TR, Acton PD, Maldjian JA, et al. Decreased gray matter concentration in the insular, orbitofrontal, cingulate, and temporal cortices of cocaine patients. Biol Psychiatry. 2002;51(2):134-142.

2. Ersche KD, Barnes A, Jones PS, Morein-Zamir S, Robbins TW, Bullmore ET. Abnormal structure of frontostriatal brain systems is associated with aspects of impulsivity and compulsivity in cocaine dependence. Brain. 2011;134(Pt 7):2013-2024.

3. Hanlon CA, Dufault DL, Wesley MJ, Porrino LJ. Elevated gray and white matter densities in cocaine abstainers compared to current users. Psychopharmacology. 2011;218(4):681-692.

4. Taylor WD, Hsu E, Krishnan KR, MacFall JR. Diffusion tensor imaging: background, potential, and utility in psychiatric research. Biol Psychiatry. 2004;55(3):201-207.

5. Lim KO, Wozniak JR, Mueller BA, et al. Brain macrostructural and microstructural abnormalities in cocaine dependence. Drug Alcohol Depend. 2008;92(1-3):164-172.

6. Romero MJ, Asensio S, Palau C, Sanchez A, Romero FJ. Cocaine addiction: diffusion tensor imaging study of the inferior frontal and anterior cingulate white matter. Psychiatry Res. 2010;181(1):57-63.

7. Xu J, DeVito EE, Worhunsky PD, Carroll KM, Rounsaville BJ, Potenza MN. White matter integrity is associated with treatment outcome measures in cocaine dependence. Neuropsychopharmacology. 2010;35(7):1541-1549.

8. Volkow ND, Fowler JS, Wang GJ, et al. Decreased dopamine D2 receptor availability is associated with reduced frontal metabolism in cocaine abusers. Synapse. 1993;14(2):169-177.

9. Ross B, Michaelis T. Clinical applications of magnetic resonance spectroscopy. Magn Reson Q. 1994;10(4):191-247.

10. Kalivas PW, Volkow N, Seamans J. Unmanageable motivation in addiction: a pathology in prefrontal-accumbens glutamate transmission. Neuron. 2005;45(5):647-650.

11. Schmaal L, Veltman DJ, Nederveen A, van den Brink W, Goudriaan AE. $\mathrm{N}$-acetylcysteine normalizes glutamate levels in cocaine-dependent patients: a randomized crossover magnetic resonance spectroscopy study. Neuropsychopharmacology. 2012;37(9):2143-2152.

12. Yang S, Salmeron BJ, Ross TJ, Xi ZX, Stein EA, Yang Y. Lower glutamate levels in rostral anterior cingulate of chronic cocaine users a (1) H-MRS study using TE-averaged PRESS at $3 \mathrm{~T}$ with an optimized quantification strategy. Psychiatry Res. 2009;174(3):171-176.

13. Mardikian PN, LaRowe SD, Hedden S, Kalivas PW, Malcolm RJ. An open-label trial of $\mathrm{N}$-acetylcysteine for the treatment of cocaine dependence: a pilot study. Prog Neuropsychopharmacol Biol Psychiatry. 2007;31(2):389-394.

14. Chang L, Ernst T, Strickland T, Mehringer CM. Gender effects on persistent cerebral metabolite changes in the frontal lobes of abstinent cocaine users. Am J Psychiatry. 1999;156(5):716-722.

15. Garavan H, Pankiewicz J, Bloom A, et al. Cue-induced cocaine craving: neuroanatomical specificity for drug users and drug stimuli. Am J Psychiatry. 2000;157(11):1789-1798.

16. Maas LC, Lukas SE, Kaufman MJ, et al. Functional magnetic resonance imaging of human brain activation during cue-induced cocaine craving. Am J Psychiatry. 1998;155(1):124-126.

17. Wexler BE, Gottschalk CH, Fulbright RK, et al. Functional magnetic resonance imaging of cocaine craving. Am J Psychiatry. 2001;158(1): 86-95.

18. Detre JA, Zhang W, Roberts DA, et al. Tissue specific perfusion imaging using arterial spin labeling. NMR Biomed. 1994;7(1-2):75-82.

19. Detre JA, Wang J. Technical aspects and utility of fMRI using BOLD and ASL. Clin neurophysiol. 2002;113(5):621-634.
20. Tjandra T, Brooks JC, Figueiredo P, Wise R, Matthews PM, Tracey I. Quantitative assessment of the reproducibility of functional activation measured with BOLD and MR perfusion imaging: implications for clinical trial design. Neuroimage. 2005;27(2):393-401.

21. Durazzo TC, Gazdzinski S, Mon A, Meyerhoff DJ. Cortical perfusion in alcohol-dependent individuals during short-term abstinence: relationships to resumption of hazardous drinking after treatment. Alcohol. 2010;44(3):201-210.

22. Jacobus J, Goldenberg D, Wierenga CE, Tolentino NJ, Liu TT, Tapert SF. Altered cerebral blood flow and neurocognitive correlates in adolescent cannabis users. Psychopharmacology (Berl). 2012;222(4): 675-684.

23. Howell LL, Murnane KS. Nonhuman primate positron emission tomography neuroimaging in drug abuse research. J Pharmacol Exp Ther. 2011;337(2):324-334.

24. Cosgrove KP. Imaging receptor changes in human drug abusers. Curr Top Behav Neurosci. 2010;3:199-217.

25. Volkow ND, Wang GJ, Fischman MW, et al. Relationship between subjective effects of cocaine and dopamine transporter occupancy. Nature. 1997;386(6627):827-830.

26. Breiter HC, Gollub RL, Weisskoff RM, et al. Acute effects of cocaine on human brain activity and emotion. Neuron. 1997;19(3): 591-611.

27. Kufahl PR, Li Z, Risinger RC, et al. Neural responses to acute cocaine administration in the human brain detected by fMRI. Neuroimage. 2005;28(4):904-914.

28. Kilts CD, Gross RE, Ely TD, Drexler KP. The neural correlates of cue-induced craving in cocaine-dependent women. Am J Psychiatry. 2004;161(2):233-241.

29. Risinger RC, Salmeron BJ, Ross TJ, et al. Neural correlates of high and craving during cocaine self-administration using BOLD fMRI. Neuroimage. 2005;26(4):1097-1108.

30. Everitt BJ, Robbins TW. Neural systems of reinforcement for drug addiction: from actions to habits to compulsion. Nat Neurosci. 2005; 8(11):1481-1489.

31. Porrino LJ, Lyons D, Smith HR, Daunais JB, Nader MA. Cocaine self-administration produces a progressive involvement of limbic, association, and sensorimotor striatal domains. J Neurosci. 2004;24(14): 3554-3562.

32. Hanlon CA, Wesley MJ, Porrino LJ. Loss of functional specificity in the dorsal striatum of chronic cocaine users. Drug Alcohol Depend. 2009;102(1-3):88-94.

33. Balleine BW, Delgado MR, Hikosaka O. The role of the dorsal striatum in reward and decision-making. $J$ Neurosci. 2007;27(31): 8161-8165.

34. Jacobsen LK, Giedd JN, Gottschalk C, Kosten TR, Krystal JH. Quantitative morphology of the caudate and putamen in patients with cocaine dependence. Ame J Psychiatry. 2001;158(3):486-489.

35. Sinha R, Lacadie C, Skudlarski P, et al. Neural activity associated with stress-induced cocaine craving: a functional magnetic resonance imaging study. Psychopharmacology. 2005;183(2):171-180.

36. Wong DF, Kuwabara H, Schretlen DJ, et al. Increased occupancy of dopamine receptors in human striatum during cue-elicited cocaine craving. Neuropsychopharmacology. 2006;31(12):2716-2727.

37. Moeller FG, Steinberg JL, Schmitz JM, et al. Working memory fMRI activation in cocaine-dependent subjects: association with treatment response. Psychiatry Res. 2010;181(3):174-182.

38. Brewer JA, Worhunsky PD, Carroll KM, Rounsaville BJ, Potenza MN. Pretreatment brain activation during stroop task is associated with outcomes in cocaine-dependent patients. Biol Psychiatry. 2008;64(11): 998-1004.

39. Sim ME, Lyoo IK, Streeter CC, et al. Cerebellar gray matter volume correlates with duration of cocaine use in cocaine-dependent subjects. Neuropsychopharmacology. 2007;32(10):2229-2237.

40. Tomasi D, Goldstein RZ, Telang F, et al. Thalamo-cortical dysfunction in cocaine abusers: implications in attention and perception. Psychiatry Res. 2007;155(3):189-201. 
41. Davis M, Whalen PJ. The amygdala: vigilance and emotion. Mol Psychiatry. 2001;6(1):13-34.

42. Childress AR, Ehrman RN, Wang Z, et al. Prelude to passion: limbic activation by "unseen" drug and sexual cues. PLoS one. 2008;3 (1):e1506.

43. Makris N, Gasic GP, Seidman LJ, et al. Decreased absolute amygdala volume in cocaine addicts. Neuron. 2004;44(4):729-740.

44. Bonson KR, Grant SJ, Contoreggi CS, et al. Neural systems and cueinduced cocaine craving. Neuropsychopharmacology. 2002;26(3): 376-386.

45. Childress AR, Mozley PD, McElgin W, Fitzgerald J, Reivich M, O’Brien CP. Limbic activation during cue-induced cocaine craving. Am J Psychiatry. 1999;156(1):11-18.

46. Kilts CD, Schweitzer JB, Quinn CK, et al. Neural activity related to drug craving in cocaine addiction. Arch Gen Psychiatry. 2001;58(4): 334-341.

47. Miller EK, Cohen JD. An integrative theory of prefrontal cortex function. Ann Rev Neurosci. 2001;24:167-202.

48. Grant S, London ED, Newlin DB, et al. Activation of memory circuits during cue-elicited cocaine craving. Proc Natl Acad Sci U S A. 1996;93(21):12040-12045.

49. Matochik JA, London ED, Eldreth DA, Cadet JL, Bolla KI. Frontal cortical tissue composition in abstinent cocaine abusers: a magnetic resonance imaging study. Neuroimage. 2003;19(3):1095-1102.

50. Kaufman JN, Ross TJ, Stein EA, Garavan H. Cingulate hypoactivity in cocaine users during a GO-NOGO task as revealed by event-related functional magnetic resonance imaging. $J$ Neurosci. 2003;23(21): 7839-7843.

51. Kubler A, Murphy K, Garavan H. Cocaine dependence and attention switching within and between verbal and visuospatial working memory. Eur J Neurosci. 2005;21(7):1984-1992.

52. Bolla KI, Eldreth DA, London ED, et al. Orbitofrontal cortex dysfunction in abstinent cocaine abusers performing a decision-making task Neuroimage. 2003;19(3):1085-1094.

53. Krawczyk DC. Contributions of the prefrontal cortex to the neural basis of human decision making. Neurosci Biobehav Rev. 2002;26(6): 631-664.

54. Makris N, Gasic GP, Kennedy DN, et al. Cortical thickness abnormalities in cocaine addiction - a reflection of both drug use and a pre-existing disposition to drug abuse? Neuron. 2008;60(1):174-188.

55. Wilcox CE, Teshiba TM, Merideth F, Ling J, Mayer AR. Enhanced cue reactivity and fronto-striatal functional connectivity in cocaine use disorders. Drug Alcohol Depend. 2011;115(1-2):137-144.

56. Volkow ND, Fowler JS. Addiction, a disease of compulsion and drive: involvement of the orbitofrontal cortex. Cereb Cortex. 2000;10(3): 318-325.

57. Kringelbach ML, Rolls ET. The functional neuroanatomy of the human orbitofrontal cortex: evidence from neuroimaging and neuropsychology. Prog Neurobiol. 2004;72(5):341-372.

58. Wilson SJ, Sayette MA, Fiez JA. Prefrontal responses to drug cues: a neurocognitive analysis. Nat Neurosci. 2004;7(3):211-214.

59. Volkow ND, Fowler JS, Wang GJ, et al. Cognitive control of drug craving inhibits brain reward regions in cocaine abusers. Neuroimage. 2010;49(3):2536-2543.

60. Bush G, Luu P, Posner MI. Cognitive and emotional influences in anterior cingulate cortex. Trends Cogn Sci. 2000;4(6):215-222.

61. Goldstein RZ, Leskovjan AC, Hoff AL, et al. Severity of neuropsychological impairment in cocaine and alcohol addiction: association with metabolism in the prefrontal cortex. Neuropsychologia. 2004;42(11): 1447-1458.

62. Goldstein RZ, Volkow ND. Drug addiction and its underlying neurobiological basis: neuroimaging evidence for the involvement of the frontal cortex. Am J Psychiatry. 2002;159(10):1642-1652.

63. Volkow ND, Fowler JS, Wolf AP, et al. Changes in brain glucose metabolism in cocaine dependence and withdrawal. Am J Psychiatry. 1991;148(5):621-626.
64. Volkow ND, Hitzemann R, Wang GJ, et al. Long-term frontal brain metabolic changes in cocaine abusers. Synapse. 1992;11(3): 184-190.

65. Volkow ND, Wang GJ, Ma Y, et al. Activation of orbital and medial prefrontal cortex by methylphenidate in cocaine-addicted subjects but not in controls: relevance to addiction. J Neurosci. 2005;25(15):3932-3939.

66. Hester R, Garavan H. Executive dysfunction in cocaine addiction: evidence for discordant frontal, cingulate, and cerebellar activity. J Neuroscience. 2004;24(49):11017-11022

67. Singer T, Critchley HD, PreuschoffK. A common role of insula in feelings, empathy and uncertainty. Trends Cogn Sci. 2009;13(8):334-340.

68. Friston KJ. Functional and effective connectivity: a review. Brain Connect. 2011;1(1):13-36.

69. Hanlon CA, Wesley MJ, Roth AJ, Miller MD, Porrino LJ. Loss of laterality in chronic cocaine users: an fMRI investigation of sensorimotor control. Psychiatry Res. 2010;181(1):15-23.

70. Hanlon CA, Wesley MJ, Stapleton JR, Laurienti PJ, Porrino LJ. The association between frontal-striatal connectivity and sensorimotor control in cocaine users. Drug Alcohol Depend. 2011;115(3): 240-243.

71. Harrison BJ, Soriano-Mas C, Pujol J, et al. Altered corticostriatal functional connectivity in obsessive-compulsive disorder. Arch Gen Psychiatry. 2009;66(11):1189-1200.

72. Jeffries KJ, Schooler C, Schoenbach C, Herscovitch P, Chase TN, Braun AR. The functional neuroanatomy of Tourette's syndrome: an FDG PET study III: functional coupling of regional cerebral metabolic rates. Neuropsychopharmacology. 2002;27(1):92-104.

73. Cao X, Cao Q, Long X, et al. Abnormal resting-state functional connectivity patterns of the putamen in medication-naive children with attention deficit hyperactivity disorder. Brain Res. 2009;1303: 195-206.

74. Gu H, Salmeron BJ, Ross TJ, et al. Mesocorticolimbic circuits are impaired in chronic cocaine users as demonstrated by resting-state functional connectivity. Neuroimage. 2010;53(2):593-601.

75. Ma N, Liu Y, Li N, et al. Addiction related alteration in resting-state brain connectivity. Neuroimage. 2010;49(1):738-744.

76. Kelly C, Zuo XN, Gotimer K, et al. Reduced interhemispheric resting state functional connectivity in cocaine addiction. Biol Psychiatry. 2011;69(7):684-692.

77. Li SJ, Biswal B, Li Z, et al. Cocaine administration decreases functional connectivity in human primary visual and motor cortex as detected by functional MRI. Magn Reson Med. 2000;43(1):45-51.

78. Bestmann S, Baudewig J, Siebner HR, Rothwell JC, Frahm J. Subthreshold high-frequency TMS of human primary motor cortex modulates interconnected frontal motor areas as detected by interleaved fMRI-TMS. Neuroimage. 2003;20(3):1685-1696.

79. Li X, Teneback CC, Nahas Z, et al. Interleaved transcranial magnetic stimulation/functional MRI confirms that lamotrigine inhibits cortical excitability in healthy young men. Neuropsychopharmacology. 2004; 29(7):1395-1407.

80. Bartzokis G, Beckson M, Lu PH, et al. Age-related brain volume reductions in amphetamine and cocaine addicts and normal controls: implications for addiction research. Psychiatry Res. 2000;98(2):93-102.

81. Fein G, Di Sclafani V, Meyerhoff DJ. Prefrontal cortical volume reduction associated with frontal cortex function deficit in 6-week abstinent crackcocaine dependent men. Drug Alcohol Depend. 2002;68(1):87-93.

82. Lim KO, Choi SJ, Pomara N, Wolkin A, Rotrosen JP. Reduced frontal white matter integrity in cocaine dependence: a controlled diffusion tensor imaging study. Bioll Psychiatry. 2002;51(11):890-895.

83. Moeller FG, Hasan KM, Steinberg JL, et al. Reduced anterior corpus callosum white matter integrity is related to increased impulsivity and reduced discriminability in cocaine-dependent subjects: diffusion tensor imaging. Neuropsychopharmacology. 2005;30(3):610-617.

84. O’Neill J, Cardenas VA, Meyerhoff DJ. Separate and interactive effects of cocaine and alcohol dependence on brain structures and metabolites: quantitative MRI and proton MR spectroscopic imaging. Addict Biol. 2001;6(4):347-361. 
85. Bartzokis G, Beckson M, Lu PH, Edwards N, Bridge P, Mintz J. Brain maturation may be arrested in chronic cocaine addicts. Biol Psychiatry. 2002;51(8):605-611.

86. Moeller FG, Hasan KM, Steinberg JL, et al. Diffusion tensor imaging eigenvalues: preliminary evidence for altered myelin in cocaine dependence. Psychiatry Res. 2007;154(3):253-258.
87. Lane SD, Steinberg JL, Ma L, et al. Diffusion tensor imaging and decision making in cocaine dependence. PLoS one. 2010;5(7):e11591.

\section{Publish your work in this journal}

Substance Abuse and Rehabilitation is an international, peer-reviewed, open access journal publishing original research, case reports, editorials, reviews and commentaries on all areas of addiction and substance abuse and options for treatment and rehabilitation. The manuscript management system is completely online and includes a very quick and fair peer-review system. Visit http://www.dovepress.com/testimonials.php to read real quotes from published authors. 